\title{
Оценка условий фильтрации через земляные плотины при изменении их параметров с использованием программы Z_SOIL
}

\author{
(C)2020 Я. ШІІ. Мохамед ${ }^{1}$ Н. А. Виноград ${ }^{1 凶}$, А. А. Потапов ${ }^{2}$

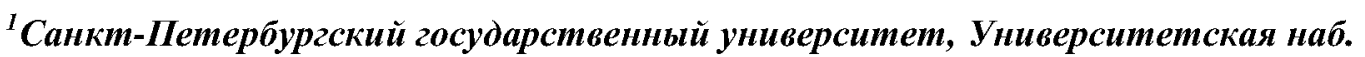 \\ 7/9, 199034, Санкт-Петербург, Российская Федерация \\ ${ }^{2}$ Горный институт Уральского отделения Российской академии наук, филиал \\ Пермского федерального исследовательского центра УрО РАН, ул. Сибирская, д. 78-А, \\ 614007, Пермский край, Пермь, Российская Федерация
}

\begin{abstract}
Аннотация
Введение: В Арабской Республике Египет (АРЕ) использование земляных плотин для предотвращения риска затопления является повсеместным. Для всех земляных плотин водохранилищ характерна фильтрация воды через их насыпные дамбы, основания и примыкающие борта долины - создается искусственный водоносный горизонт. Количественная оценка данного процесса сама по себе является серьёзной гидрогеологической задачей, а также необходима для решения многих гидрологических и геоэкологических проблем.

Mетодика: Использованная для численного решения задачи программа Z-SOIL реализована на основе метода конечных элементов. Программа предназначена для моделирования неустановившейся фильтрации с возможностью варьирования уровнями воды, размерами фильтрующего объекта и свойствами слагающих его грунтов. В начале исследования эксперимент по моделированию фильтрации и проницаемости грунта был проведен на физической модели плотины в лабораторных условиях. Затем проводилась калибровка численной геофильтрационной модели путем сравнения модельных и экспериментальных данных. И, наконец, оценивалось влияние изменения геометрии плотины на уровень воды в водохранилище.

Результаты и обсуждение: Результаты численного моделирования хорошо согласуются с экспериментальными данными. Было получено, что по мере увеличения ширины гребня плотины и высоты основания уровни воды в водохранилище за счет фильтрации через плотину будут уменьшаться с максимальным изменением во времени до $0.3 \%$ и $0.26 \%$, соответственно.

$B b l 6 o \partial b l:$ Используя степень насыщения горных пород и напоры на верхнем бьефе водохранилища в качестве переменных, модель Z-SOIL позволяет моделировать неустановившуюся фильтрацию через земляную плотину. Сравнение численных решений с экспериментальными результатами показывает, что описанный способ моделирования позволяет довольно точно имитировать изменения уровенной поверхности внутри плотины (насыпной дамбы) во времени в реальных условиях. Результаты хорошо согласуются с практикой эксплуатации песчаных плотин и позволяют осуществить предварительную оценку фильтрации через плотину и подбор параметров при строительстве.
\end{abstract}

Ключевые слова: подземные воды; поверхностные воды; нестационарная фильтрация; численная модель геофильтрации; земляная плотина; насыпная дамба водохранилища; метод конечных элементов.

Контент доступен под лицензией Creative Commons Attribution 4.0 License.

\footnotetext{
冈 Виноград Наталия Анатольевна, e-mail: nv.70@hotmail.com
} 
Для ичитирования: Мохамед Я. Ш., Виноград Н. А., Потапов А. А. Оценка условий фильтрации через земляные плотины при изменении их параметров с использованием программы Z_SOIL // Вестник Воронежского государственного университета. Серия: Геология. 2020. № 2. С. 90-97. DOI: https://doi.org/10.17308/geology.2020.2/2863

\section{Введение}

В Арабской Республике Египет использование земляных плотин для предотвращения риска затопления является повсеместным. Такая конструкция при невысокой стоимости служит резервуаром для водных масс, предотвращая испарение и при необходимости позволяя дальнейшее использование воды, накапливающейся в теле плотины, путем бурения скважин или строительства дренажных отводов в теле плотины. Как правило, земляные плотины в АРЕ строятся из однородного местного материала (песок) и не имеют сердечника, а также фильтров и сливных зон. При подъеме уровня воды происходит фильтрация в тело плотины, и образуется искусственный водоносный горизонт. Изучение условий и параметров фильтрации воды через насыпные дамбы плотин водохранилищ в нестационарных граничных условиях (то есть в искусственно созданных водоносных горизонтах со специфическими граничными условиями) само по себе является серьёзной гидрогеологической задачей.

Фильтрация через насыпную дамбу, основание и примыкающие борта долины характерна для всех земляных плотин. Ее контроль необходим для предотвращения избыточного давления, осыпания склона в нижнем бьефе, просадки насыпи и основания, а также эрозии материала и потерь в открытые трещины в основании и устоях [1].

Кроме того, рассмотрение этой задачи представляет интерес и для решения многих других сопутствующих гидрологических и геоэкологических проблем, таких как регулирование объемов и химического состава стока подземных вод, обеспечение устойчивости земляных плотин и склонов водохранилищ при высокой эрозионности песков и изменяющихся уровнях воды в них, оценка гидрогеодинамического и гидрогеохимического взаимодействия поверхностных и подземных вод при регулировании водных ресурсов, загрязнение подземных и поверхностных вод, а также влияние возможных интрузий морской воды в водоносные горизонты в прибрежных районах [2] и др.

Цель данной работы - обосновать возможность использования программного средства Z-Soil версии 5.79 для численного моделирования фильтрации через песчаную плотину.

В рамках работы решались следующие задачи:

1. Создание экспериментальной модели типичной песчаной плотины с возможностью фиксации уровней воды в теле плотины.
2. Описание строения и размеров экспериментальной модели с помощью программного средства Z-Soil версии 5.79.

3. Проведение модельных экспериментов по подъему уровня воды в верхнем бьефе с регистрацией положения фильтрационной поверхности в теле плотины с помощью 27 пьезометров.

4. Описание эксперимента численными методами средствами Z-Soil 5.79 и калибровка модели путем сопоставления численных и экспериментальных данных.

5. Применение программного средства Z-Soil версии 5.79 для моделирования нестационарной фильтрации через песчаную плотину с изменением параметров плотины. Оценка выводов.

\section{Методика}

Научный задел. Создавать численные модели реальных объектов довольно сложно из-за разнородности природных грунтов и изменчивости граничных условий [3]. Ли и др. [4] проанализировано влияние циклов засух-наводнений на фильтрацию в земляной дамбе экспериментально и методом конечных элементов при нестационарном режиме. Показано, что долгосрочная засуха вызывает глубокие трещины и резкое увеличение всасывающего давления, т.е. трещины станут каналами инфильтрации атмосферных осадков в плотину, увеличивая потенциал для внутренней эрозии и снижая стабильность плотины.

Тестирование и верификация одиночной и интегральной модели ИНС (искусственной нейронной сети) пьезометрических напоров на примере ядра насыпной плотины Саттархан в Иране продемонстрировали хорошую сходимость между вычисленными и наблюдаемыми уровнями воды [5]. Анализ одномерной стационарной модели фильтрации в ненасыщенных грунтах в вакууме показал, что наличие вакуума значительно повышает дренирующую способность ненасыщенного грунта за счет увеличения гидравлического градиента [6]. Результаты анализа стационарной и нестационарной фильтрации и стабильности земляной плотины с использованием метода конечных элементов показали, что увеличение модуля Юнга ядра и тела плотины привело к уменьшению максимального смещения гребня, а изменение угла внутреннего трения играет важную роль в выполнении требований общей устойчивости [3].

Для описания и экспериментальной проверки процесса фильтрации Юнь-Фень и др. [2] предложили численную модель неустановившегося фильтрационного потока через плотину, с переменными степенью насыщения и уровнем. Показана возможность применения численных методов расчета депрессионной кривой через описание степени насыщения. Джамель и др. [7] использовали программное средство SEEP/W для разработки эмпирического уравнения с целью определения величины фильтрации через однородную земляную плотину. Верификация результатов показала, что предложенное уравнение дает ошибку 2-3\%. При этом 
решение Дюпюи дает ошибку более чем $20 \%$, а решение Касагранде - более чем $15 \%$.

При изучении проблемы фильтрации имеют значение следующие характеристики: основание земляной плотины, УВБ (уровень верхнего бьефа), УНБ (уровень нижнего бьефа), свободная поверхность воды в земляной плотине, разность УНБ и свободной поверхности в нижнем бьефе плотины, фильтрационная поверхность и расход двумерного потока через произвольное поперечное сечение земляной плотины [8].

В APE широко применяются плотины с песчаноземляным заполнением. Этот тип плотины характеризуется низкой стоимостью вследствие легкой доступности природных материалов, а также быстроты и простоты строительства. Такие плотины используются в различных целях, таких как защита от наводнений [4], добыча полезных ископаемых (в качестве хвостохранилища) [9] и временное сооружение вокруг участка строительства, чтобы предотвратить попадание воды на этот участок до завершения работ. Различные параметры, влияющие на процесс фильтрации через плотину и вид фильтрационной поверхности внутри плотины, а также долгосрочные модели деформации об- суждаются в работах $[10,11]$.

При проектировании и строительстве плотин необходимо обращать внимание на экологическую составляющую, в частности, проводить оценку геоэкологических рисков и последствий техногенного изменения ландшафтов $[12,13]$. Эта проблема, безусловно, является актуальной и для АРЕ, где условиях аридного климата при строительстве подобных объектов происходят особенно заметные изменения естественного гидрологического и гидрогеологического режимов.

Экспериментальная модель. Цель создания экспериментальной модели - проверка пригодности численной модели Z-Soil для моделирования двумерной задачи со сложными граничными условиями. В лабораторных условиях была создана модель песчаной плотины, наиболее часто применяемой для регулирования речного стока в АРЕ, в частности, на Синайском полуострове.

Работа проводилась в Лаборатории гидравлики и механики флюидов факультета Инжиниринга, университета г. Загазиг, Египет. Схема экспериментальной установки и ее размеры показаны на рис. 1.

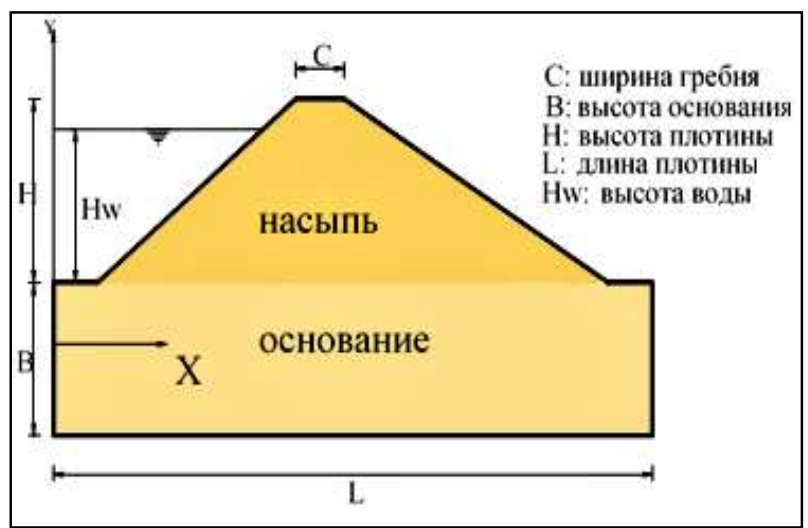

a

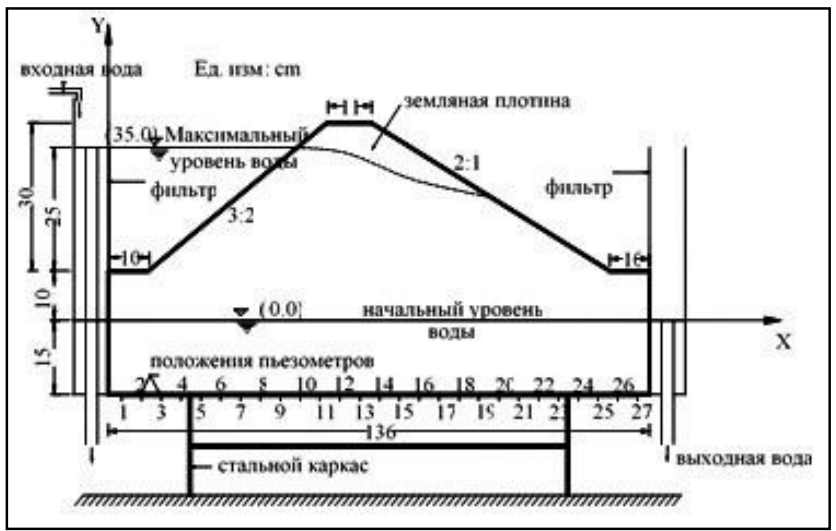

b

Рис. 1. Строение (a) и размеры (b) экспериментальной модели плотины (поперечный разрез плотины, вдоль потока). [Fig. 1. Structure (a) and dimensions (b) of the experimental dam model (cross section of the dam, along the stream).]

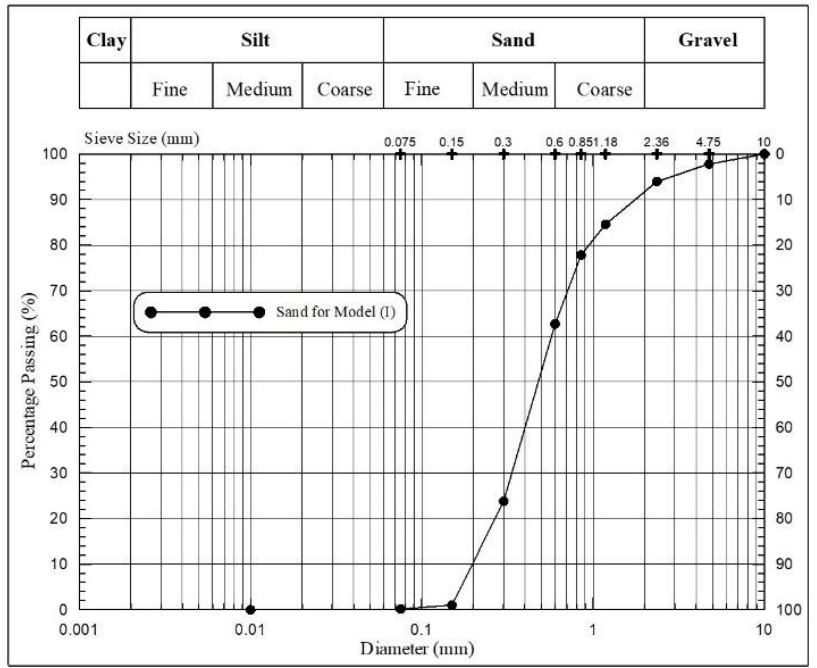

Для создания модели использовался один тип грунта - песок, гранулометрический состав которого представлен на рис. 2. Коэффициент пористости оценивается в 0.23. Коэффициент фильтрации в зоне насыщения определен в условиях стационарной фильтрации, $\mathrm{K}=3.5 \cdot 10^{-4} \mathrm{M} / \mathrm{c}$ (около $30 \mathrm{M} / \mathrm{сут)}$ ).

Численная модель. В настоящей работе методом конечных элементов решается задача фильтрации через плотину с использованием программы Z-Soil версии 5.79. Описывалась созданная экспериментальная модель.

На рис. 3 показана рабочая область программы и прямоугольная сетка с заданием фильтрационных параметров и свойств грунтов для всех элементов сетки.

Рис. 2. Гранулометрический состав песка.

[Fig. 2. Granulometric composition of sand.] 

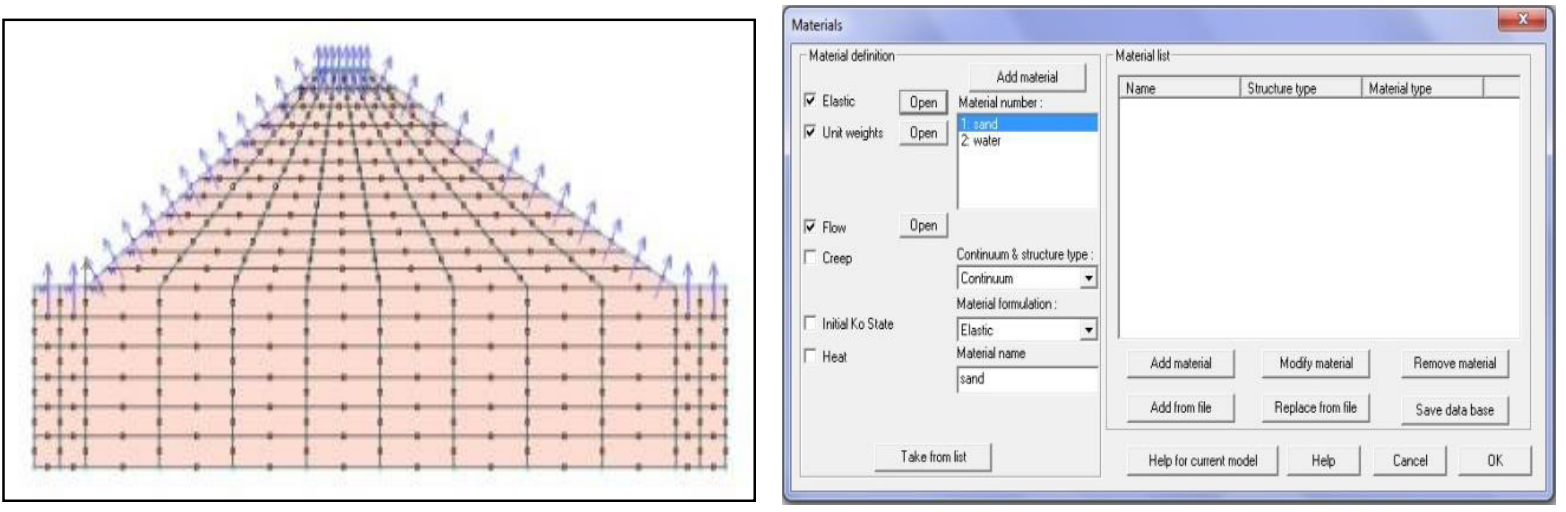

Рис. 3. Задание сетки и интерфейс рабочей области программы.

[Fig. 3. Grid setting and the interface of the software workspace.]
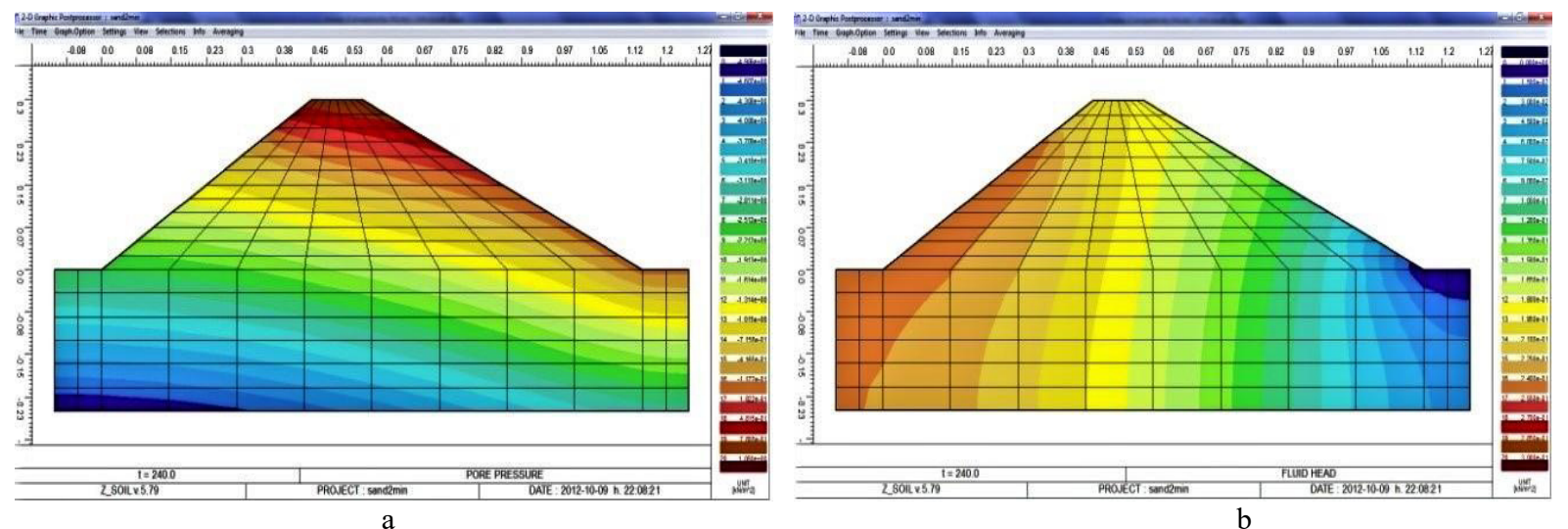

Рис. 4. Выходные данные: $a$ - распределение давления поровой воды; $b$-распределение напоров.

[Fig. 4. Output data: $a$-pore water pressure distribution; $b$ - head distribution.]

Изучаемый объект представляет собой упрощенную модель типичной песчаной плотины. Для песка использованы следующие параметры: модуль упругости $(\mathrm{E})=10^{4} \kappa г / \mathrm{M}^{2} ;$ коэффициент Пуассона $(\mathrm{v})=0.3$;

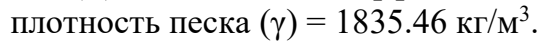

На рис. 4 показаны выходные данные - давление поровой воды (a) и распределение гидравлического напора (b) в виде цветных карт.

Ход эксперимента. В начальных условиях в верхнем бьефе вода отсутствует. Затем уровень воды поднимется от 0 до 0.35 м за 4 ч. Давление в каждой точке пьезометра (рис. 1 б) регистрировалось с интервалом в 15 мин. в течение первого часа и 30 мин. в остальные 3 часа. Положение фильтрационной поверхности регистрировалось через те же интервалы.

Сравнение экспериментальных и численных данных показано на рис. 5. Высота плотины $(\mathrm{H}=0.3$ м) была использована в качестве реферативной точки. На рис. 5а показано положение фильтрационной поверхности в разрезе через указанные интервалы времени 15 мин., 45 мин., 1.5 ч, 2.5 ч и 4 ч (красными точками экспериментальные результаты, черной линией - результаты численного моделирования). Рисунок 56 показывает распределение уровней воды в плотине в разных точках пьезометра $(1,7,12,16,20,23,25,27)$. Крестиками показаны экспериментальные результаты, черными линиями - результаты численного моделиро- вания. Как видно на рисунке, численные решения хорошо согласуются с экспериментально полученными данными. Это показывает, что численная модель может быть использована для исследования фильтрационной поверхности при неустановившейся фильтрации через плотину.

\section{Обсуждение результатов}

Как показано на рис. 5, результаты численного моделирования с помощью программы Z_SOIL хорошо согласуются с экспериментальными данными. Эта программа может использоваться для расчета неустановившегося уровня и исследования фильтрационной поверхности при фильтрации через земляную плотину.

Исследование нестационарного потока через земляную плотину включает построение депрессионной кривой. Можно изменять некоторые параметры, влияющие на градиент фильтрационного потока, такие как ширина гребня $(C)$, высота основания $(B)$, отношение горизонтальных и вертикальных коэффициентов фильтрации основания и насыпи $\left(k_{x} / k_{y}\right)$, а затем сравнить напоры на каждом пьезометре с экспериментальной моделью, т.е. $C=12 \mathrm{~cm}, B=24 \mathrm{~cm}, k_{x} / k_{y}=1$. Для упрощения использовались усредненные данные 10 пьезометров, представляющие также соседние точки (всего имелось 27 пьезометров). 

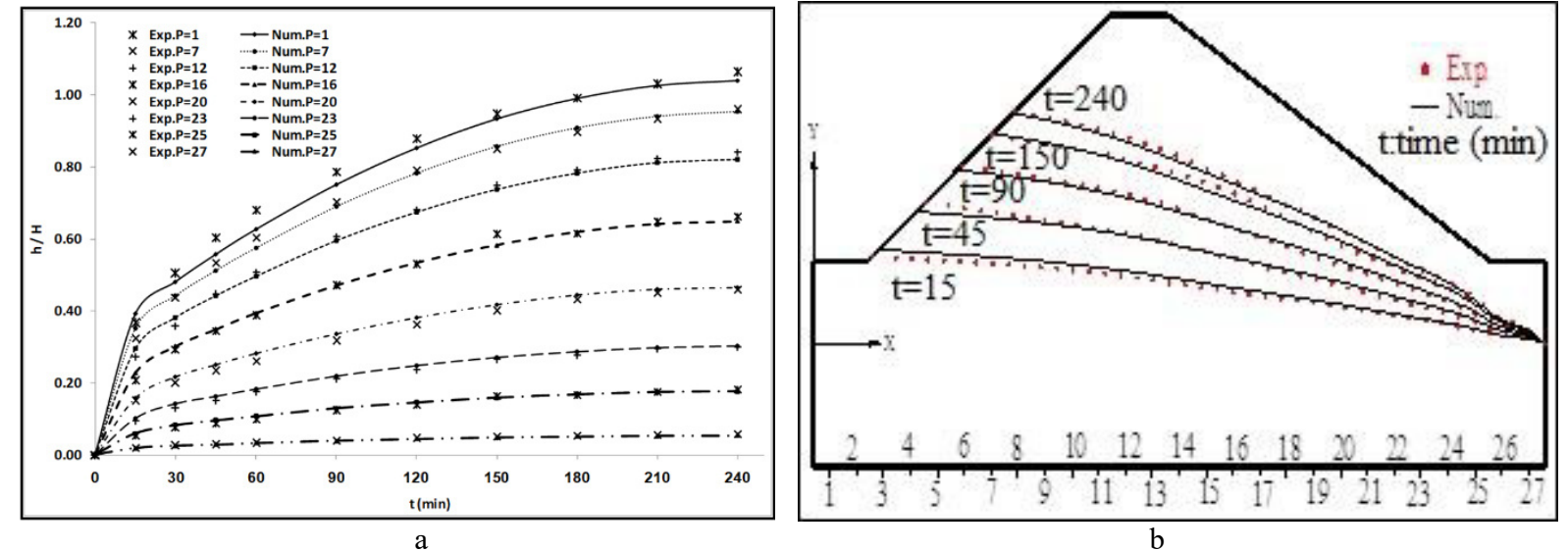

Рис. 5. Сравнение результатов численного и экспериментального моделирования. На рис. 5а красными точками показаны экспериментальные результаты, черной линией - результаты численного моделирования. На рис. $5 \mathrm{~b}$ крестиками показаны экспериментальные результаты, черными линиями - результаты численного моделирования. $H$ - высота плотины, $h$ - мощность фильтрационного потока.

[Fig. 5. Comparison of numerical and experimental modeling results. Figure 5a shows experimental results (with red dots) and numerical simulation results (with a black line). Figure $5 \mathrm{~b}$ shows experimental results with crosses, and numerical simulation results with black lines. $H$ is the height of the dam, $h$ is the height of the groundwater flow.]

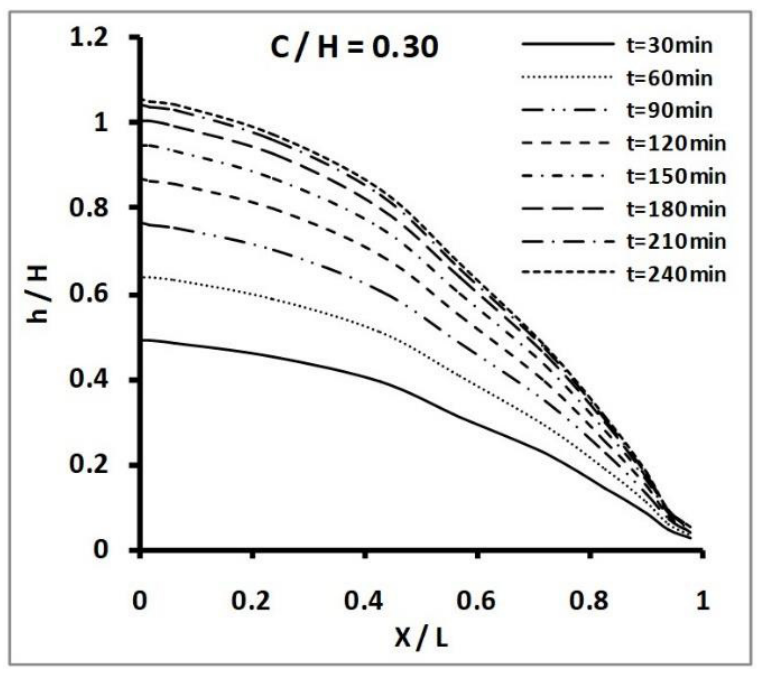

a

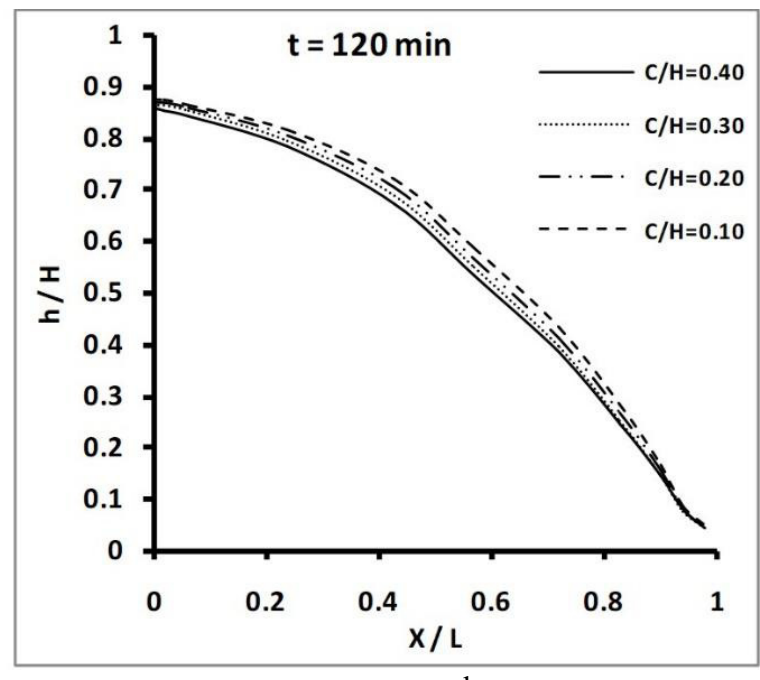

$\mathrm{b}$

Рис. 6. Сравнение напоров на разные моменты времени (a) и с различной шириной гребня C (b).

[Fig. 6. Comparison of heads at different time points (a) and with different crest widths $\mathrm{C}$ (b).]

В первом эксперименте изменялась ширина гребня $(C)$, принимались значения $12 \mathrm{~cm}, 9 \mathrm{~cm}, 6$ см и 3 см с постоянными высотой основания $(B)$ и отношением горизонтальных и вертикальных коэффициентов фильтрации основания и насыпи $\left(k_{x} / k_{y}\right)$ (рис. 6).

Во втором эксперименте высота основания $(B)$ принималась 30 см, 24 см и 15 см с постоянной шириной гребня $(C)$, отношением горизонтальных и вертикальных коэффициентов фильтрации основания и насыпи $\left(k_{x} / k_{y}\right)$ которое, как и в первом случае, было равно 1. Наблюдалось изменение напора воды по длине плотины $L$ и во времени $t$.

В третьем эксперименте изменялись отношения горизонтального и вертикального коэффициентов фильтрации основания $\left(k_{x} / k_{y}\right)-1,2,4,6,8,10$ при постоянных ширине гребня $(C)$, высоте основания $(B)$ и отно- шении горизонтального и вертикального коэффициентов фильтрации насыпи $\left(k_{x} / k_{y}\right)$. Наблюдалось изменение напора воды по ширине плотины $L$ (вдоль потока) и во времени $t$.

В четвертом эксперименте изменялись отношения горизонтального и вертикального коэффициентов фильтрации насыпи $\left(k_{x} / k_{y}\right)-1,2,4,6,8,10$, при этом сохранялись постоянными ширина гребня $(C)$, высота основания $(B)$ и отношения горизонтального и вертикального коэффициентов фильтрации основания $\left(k_{x} / k_{y}\right)$, равные 1 , как в исходной модели, и наблюдалось изменение напора воды по длине плотины $L$ и во времени $t$.

Bсе четыре эксперимента показали, что градиент фильтрационного потока увеличивается со временем и подъемом уровня воды (рис. 6а). Максимальное изменение наблюдалось у верхнего бьефа 
(0.003; 0.0026; 0.0026; 0.0027, соответственно). Далее по потоку через плотину мощность фильтрационного потока $(h / H)$ уменьшалась и становилась минимальной в последней четверти плотины, линии тока сходились, и изменение напора во времени уменьшалось до нуля. Экспериментами было установлено, что увеличение ширины гребня, высоты основания, отношения горизонтального и вертикального коэффициентов фильтрации как основания, так и насыпи $\left(k_{x} / k_{y}\right)$, уменьшают напор через плотину. Для второго и четвертого экспериментов в середине графика, а для третьего - в последней четверти графика эта зависимость трансформируется в обратную, что может быть связано с увеличением площади фильтрационной поверхности.

Полученные результаты хорошо согласуются с практикой строительства и эксплуатации песчаных плотин [2, 9, 14, 15, 16, 17], поэтому численная модель Z-SOIL может быть использована для проектирования и строительства.

\section{Выводы}

Используя степень насыщения горных пород и напоры на верхнем бьефе водохранилища в качестве переменных, модель Z-SOIL позволяет моделировать неустановившуюся фильтрацию через земляную плотину. Сравнение численных решений с экспериментальными результатами показывает, что описанный способ моделирования позволяет довольно точно имитировать изменения уровенной поверхности внутри плотины (насыпной дамбы) во времени в реальных условиях.

Из анализа полученных результатов можно сделать следующие выводы:

1. Доказано, что численная модель Z-SOIL с высокой степенью точности позволяет моделировать неустановившуюся фильтрацию через земляную плотину.

2. По результатам численного моделирования увеличение ширины гребня и высоты основания плотины, а также увеличение отношения горизонтального и вертикального коэффициентов фильтрации основания и насыпи $\left(k_{x} / k_{y}\right)$ плотины значительно уменьшают среднюю величину градиента напора подземных вод в теле плотины. При этом в случае нестационарной фильтрации скорости изменения напоров фильтрующихся вод уменьшаются по длине пути фильтрации, т.е. максимальные изменения наблюдаются в верхнем бьефе.

3. Данные выводы хорошо согласуются с практикой эксплуатации песчаных плотин и позволяют осуществить предварительную оценку фильтрации через плотину и подбор параметров при строительстве.

Конфликт интересов: Авторы декларируют отсутствие явных и потенциальных конфликтов интересов, связанных с публикацией настоящей статьи.

\section{ЛИТЕРАТУРА}

1. Williams A. E. Engineering and Design Seepage Analysis and Control for Dams. Department of the Army U.S. Army Corps of Engineers. Washington, DC 20314-1000, EM 1110-2-1901. 1993.
2. Jun-Feng F. U., Sheng J. A Study on Unsteady Seepage Flow through Dam // Elsevier journal of hydrodynamics. 2009. Vol. 04. No. 21. Pp. 499-504. DOI: 10.1016/S1001-6058(08)60176-6.

3. Athani S. S., Shivamanth, Solanki C.H., Dodagoudar G.R. Seepage and Stability Analyses of Earth Dam Using Finite Element Method // Elsevier Journal of Aquatic Procedia. 2015. Vol. 04. Pp. 876-883. DOI: 10.1016/j.aqpro.2015.02.110.

4. Li Z. Ye W., Marence M., Bricker J. Unsteady Seepage Behavior of an Earthfill Dam During Drought-Flood Cycles // Journal Geosciences. 2018. Vol. 09. No. 17. DOI: 10.3390/geosciences9010017/ 5. Nourani V., Sharghi E., Aminfar M.H. Integrated ANN model for earthfill dam's seepage analysis: Sattarkhan Dam in Iran // Journal of Artificial Intelligence Research. 2012. Vol. 1. No. 2. Pp. 2237. DOI: $10.5430 /$ air.v1n2p22.

6. Huang F., Lyu J., Wang G., Liu H. One-Dimensional Vacuum Steady Seepage Model of Unsaturated Soil and Finite Difference Solution // Journal Hindawi of Mathematical Problems in Engineering. 2017, ID 9589638, 7 p. DOI: https://doi.org/10.1155/2017/9589638 7. Jamel A. A. Analysis and Estimation of Seepage through Homogenous Earth Dam without Filter // Diyala Journal of Engineering Sciences. Vol. 09. No. 02, 2016. Pp. 38-94. URL: https://www.iasj.net/ iasj?func=article\&aId=113108 (дата обращения 30.01.2020).

8. Gobeil A. Steady State Seepage through a Homogeneous Isotropic Earth Dam with Vertical Sides Overlying an Impermeable Boundary. Thesis for M.Sc., Department of Geology and Geophysics, The Graduate School of Arts and Sciences, Boston College, UMI Microform 1431268. 2005. URL: https://search.proquest.com/openview/691e45807ceac62fb93457c8ade6322b/1?pqorigsite $=$ gscholar\&cbl=18750\&diss=y (дата обращения 30.01.2020). 9. Valenzuela L. Design, construction, operation and the effect of fines content and permeability on the seismic performance of tailings sand dams in Chile // Obras y Proyectos. 2016. Vol. 19. pp. 6-22. URL: $\quad$ http://200.14.96.130/index.php/obrasyproyectos/article/view/116 (дата обращения 30.01.2020).

10. Michalis P., Pytharouli S. I. Long-term Deformation Patterns of Earth-fill Dams based on Geodetic Monitoring Data: the Pournari I Dam Case Study. $3^{\text {rd }}$ Joint International Symposium on Deformation Monitoring, Vienna, 2016. Pp. 1-5. URL: http://www.academia.edu/download/42839309/JISDM2016_Michalis_et_al.pdf (дата обращения 30.01.2020).

11. Xu, Y., Zhang Li M. Breaching Parameters for Earth and Rockfill Dams // Journal of Geotechnical and Geoenvironmental Engineering. 2009. Vol. 135. Issue 12, Pp. 1957-1970. DOI: 10.1061/ASCE_GT.1943-5606.0000162.

12. Косинова И. И., Кустова Н. Р. Теория и методология геоэкологических рисков // Вестник Воронежского государственного университета. Серия: Геология. 2008. № 2. С. 189-197.

13. Косинова И. И., Базарский О. В., Козинцев С. Н. Методика геоэкологической биоиндикации георисков техногенно-трансформированных территорий // Геориск. 2012. № 3. С. 22-25.

14. Nimbalkar S.S., Borole S. T. , Kale S., Mungase S. Slope Stability Analysis with Geo5 Software for Malin Landslide in Pune (Maharashtra) // Global Journal of Engineering Science and Researches, 2016. Pp. 9-16. URL: http://www.gjesr.com/Issues\%20PDF/TECHNOPHILIA-2016\%20-\%20Jaihind\%20Polytechhnic, $\% 20 \mathrm{Ku}-$ ran,\%20February,\%202016/3.pdf (дата обращения 30.01.2020).

15. Szczesny J., Truty A. Dam surveillance and maintenance - general approach and case studies, 2004. Pp. 1-13. URL: http://www.symposcience.eu/exl--doc/colloque/ART-00001147.pdf (дата обращения 30.01.2020).

16. Xu Y. Q., Unami K., Kawachi T. Optimal hydraulic design of earth dam cross section using saturated-unsaturated seepage flow model // Journal Advances in Water Resources, 2003. Vol. 26, Pp. 1-7.

17. Carling P., Villanueva I., Herget J., Wright N., Borodavko P., Morvan H. Unsteady 1D and 2D hydraulic models with ice dam break for Quaternary mega-flood, Altai Mountains, southern Siberia // Journal Global and Planetary Change, 2009. Pp. 24-34. DOI: 10.1016/j.gloplacha.2009.11.005. 


\title{
Assessment of the filtration through earth dams when changing their parameters using the $Z_{-}$SOIL software
}

\author{
C2020 Ya. Sh. Mohamed ${ }^{1}$, N. A. Vinograd ${ }^{1 凶}$, A. A. Potapov ${ }^{2}$ \\ ${ }^{I}$ St. Petersburg State University, 7/9 Universitetskaya naberezhnaya, \\ Saint-Petersburg 199034, Russian Federation \\ ${ }^{2}$ Mining Institute of the Ural Branch of the Russian Academy of Sciences, branch of Perm Federal \\ Research Centre of UB RAS, 78-A Sibirskaya st., Perm 614007, Russian Federation
}

\begin{abstract}
Introduction: In the Arab Republic of Egypt (ARE), earth dams are widely used to prevent flooding. All earth dams of reservoirs are typically characterised by water filtration through their embankment dams, bases, and adjoining sides of the valley, which form an artificial aquifer. A quantitative assessment of this process is a serious hydrogeological task, and it is also necessary to solve many hydrological and geoecological problems.

Methodology: The Z-SOIL program was used for the numerical solution of the issue. It is based on the finite element method. The program is designed for the simulation of non-steady state filtration with the possibility of changing water level, the size of the filtering object, and the properties of its soils. At the beginning of the study, an experiment simulating filtration and soil permeability was carried out on a physical model of a dam in the laboratory. Then the numerical geofiltration simulation was calibrated comparing the simulation and the experimental data. Finally, the impact of changes in the dam geometry on the water level in the reservoir was assessed.

Results and discussion: The results of the numerical simulation are in good agreement with the experimental data. It was found that as the width of the dam crest and the height of its base increased, the water level in the reservoir decreased due to the filtration through the dam with a maximum change in time to $0.3 \%$ and $0.26 \%$, respectively.

Conclusions: Using the degree of saturation of the rocks and the pressure at the upper pool of the reservoir as variables, the Z-SOIL model allows for the simulation of non-steady filtration through an earth dam. Comparison of numerical data with the experimental results shows that the described simulation method allows us to simulate quite accurately the changes in the surface level inside the dam (embankment dam) over time under real conditions. The results are coherent with the practice of operating sand dams and allow for a preliminary assessment of filtration through a dam and selection of the parameters for its construction. Keywords: groundwater, surface water, non-steady state filtration, numerical simulation of geofiltration, earth dam; reservoir embankment dam; finite element method.
\end{abstract}

For citation: Mohamed Ya. Sh., Vinograd N. A., Potapov A. A. Assessment of the filtration through earth dams when changing their parameters using the Z_SOIL software. Vestnik Voronezhskogo gosudarstvennogo universiteta. Seriya: Geologiya = Proceedings of Voronezh State University. Series: Geology. 2020. No. 2. pp. 90-97. DOI: https://doi.org/10.17308/geology.2020.2/2863

Conflict of interests: The authors declare the absence of obvious and potential conflicts of interest related to the publication of this article)

The content is available under Creative Commons Attribution 4.0 License.

\footnotetext{
${ }^{凶}$ Natalia A. Vinograd, e-mail: nv.70@hotmail.com
} 


\section{REFERENCES}

1. Williams A. E. Engineering and Design Seepage Analysis and Control for Dams. Department of the Army U.S. Army Corps of Engineers. Washington, DC 20314-1000, EM 1110-21901. 1993.

2. Jun-Feng F. U., Sheng J. A Study on Unsteady Seepage Flow through Dam. Elsevier journal of hydrodynamics, 2009, vol. 4, no. 21, pp. 499-504. DOI: 10.1016/S1001-6058(08)60176-6.

3. Athani S. S., Shivamanth, Solanki C.H., Dodagoudar G.R. Seepage and Stability Analyses of Earth Dam Using Finite Element Method. Elsevier Journal of Aquatic Procedia. 2015, vol. 4, pp. 876-883. DOI: 10.1016/j.aqpro.2015.02.110.

4. Li Z. Ye W., Marence M., Bricker J. Unsteady Seepage Behavior of an Earthfill Dam During Drought-Flood Cycles. Journal Geosciences. 2018, vol. 9, no. 17. DOI: 10.3390/geosciences $9010017 /$

5. Nourani V., Sharghi E., Aminfar M.H. Integrated ANN model for earthfill dam's seepage analysis: Sattarkhan Dam in Iran. Journal of Artificial Intelligence Research, 2012, vol. 1, no. 2. Pp. 22-37. DOI: 10.5430/air.v1n2p22.

6. Huang F., Lyu J., Wang G., Liu H. One-Dimensional Vacuum Steady Seepage Model of Unsaturated Soil and Finite Difference Solution. Journal Hindawi of Mathematical Problems in Engineering. 2017, ID 9589638, 7 p. DOI: https://doi.org/10.1155/2017/9589638

7. Jamel A. A. Analysis and Estimation of Seepage through Homogenous Earth Dam without Filter. Diyala Journal of Engineering Sciences, 2016, vol. 9, no. 2, pp. 38-94. URL: https://www.iasj.net/iasj?func=article\&aId=113108 (дата обращения 30.01.2020).

8. Gobeil A. Steady State Seepage through a Homogeneous Isotropic Earth Dam with Vertical Sides Overlying an Impermeable Boundary. Thesis for M.Sc., Department of Geology and Geophysics, The Graduate School of Arts and Sciences, Boston College, UMI Microform 1431268. 2005. URL: https://search.proquest.com/openview/691e45807ceac62fb93457c8ade6322b/1?pqorigsite $=$ gscholar\&cbl $=18750 \&$ diss $=y \quad$ (дата обращения 30.01.2020).

9. Valenzuela L. Design, construction, operation and the effect of fines content and permeability on the seismic performance of tailings sand dams in Chile. Obras y Proyectos. 2016, vol. 19, pp.
6-22. URL: http://200.14.96.130/index.php/obrasyproyectos/article/view/116 (дата обращения 30.01.2020).

10. Michalis P., Pytharouli S. I. Long-term Deformation Patterns of Earth-fill Dams based on Geodetic Monitoring Data: the Pournari I Dam Case Study. $3^{\text {rd }}$ Joint International Symposium on Deformation Monitoring, Vienna, 2016, pp. 1-5. URL: http://www.academia.edu/download/42839309/JISDM2016

Michalis_et_al.pdf (дата обращения 30.01.2020).

11. Xu, Y., Zhang Li M. Breaching Parameters for Earth and Rockfill Dams. Journal of Geotechnical and Geoenvironmental Engineering. 2009, vol. 135, I. 12, pp. 1957-1970. DOI: 10.1061/_ASCE_GT.1943-5606.0000162.

12. Kosinova I. I., Kustova N. R. Teoriya i metodologiya geoekologicheskikh riskov [Theory and methodology of geoecological risks]. Vestnik Voronezhskogo gosudarstvennogo universiteta. Seriya: Geologiya. = Proceedings of Voronezh State University. Series: Geology, 2008, no 2, pp. 189-197 (In Russ.)

13. Kosinova I. I., Bazarskii O. V., Kozintsev S. N. Metodika geoekologicheskoi bioindikatsii georiskov tekhnogenno-transformirovannykh territorii [Methodology of the geoecological bioindication of georisks of the technogeneously-transformed territories]. Georisk = Georisk. 2012, no 3, pp. 22-25 (In Russ.)

14. Nimbalkar S.S., Borole S. T., Kale S., Mungase S. Slope Stability Analysis with Geo5 Software for Malin Landslide in Pune (Maharashtra). Global Journal of Engineering Science and Researches, 2016. pp. 9-16. URL: http://www.gjesr.com/Issues\%20PDF/TECHNOPHILIA-2016\%20-\%20Jaihind\%20Polytechhnic,\%20Kuran,\%20February,\%202016/3.pdf (дата обращения 30.01.2020).

15. Szczesny J., Truty A. Dam surveillance and maintenance general approach and case studies, 2004, pp. 1-13. URL: http://www.symposcience.eu/exl--doc/colloque/ART00001147.pdf (дата обращения 30.01.2020).

16. Xu Y. Q., Unami K., Kawachi T. Optimal hydraulic design of earth dam cross section using saturated-unsaturated seepage flow model. Journal Advances in Water Resources, 2003, vol. 26, Pp. 1-7.

17. Carling P., Villanueva I., Herget J., Wright N., Borodavko P., Morvan H. Unsteady 1D and 2D hydraulic models with ice dam break for Quaternary mega-flood, Altai Mountains, southern Siberia. Journal Global and Planetary Change, 2009. pp. 24-34. DOI: 10.1016/j.gloplacha.2009.11.005.
Мохамед Яссер Эл Сайед Шаабан - аспирант, Санкт-Петербургский государственный университет, Санкт-Петербург, PФ; E-mail: yasershaban122.a@gmail.com ORCID http://orcid.org/0000-0002-3114-132X

Виноград Наталия Анатольевна - к. г.-м. н., доцент, СанктПетербургский государственный университет, Санкт-Петербург, Российская Федерация; E-mail: nv.70@hotmail.com ORCID http://orcid.org/0000-0002-6730-5453

Потапов Александр Анатольевич -к. г.-м. н., зав. сектором гидрогеологии, Горный институт Уральского отделения Российской академии наук, филиал Пермского федерального исследовательского центра УрО РАН, Пермь, РФ E-mail: potapov@dhspb.ru ORCID http://orcid.org/0000-0003-4255-766X

Авторы прочитали и одобрили окончательный вариант рукописи.
Mohamed, Yasser El Sayed Shaaban - post-graduate student, Saint Petersburg State University, Saint Petersburg, Russian Federation. E-mail: yasershaban122.a@gmail.com ORCID http://orcid.org/0000-0002-3114-132X

Natalia A. Vinograd - PhD in Geol.-Min., Associate Professor, Saint Petersburg State University, Saint Petersburg, Russian Federation; E-mail: nv.70@hotmail.com ORCID http://orcid.org/0000-0002-6730-5453

Alexander A. Potapov - PhD in Geol.-Min., Head of the sector of Hydrogeology. Mining Institute of the Ural branch of the Russian Academy of Sciences, branch of the Perm Federal research center Uro RAS, Perm, Russian Federation

E-mail: potapov@dhspb.ru ORCID http://orcid.org/0000-0003-4255-766X

All authors have read and approved the final manuscript. 